Series A

I. MATHEMATICA

$336 / 1$

ÜBER DAS ANWACHSEN EINIGER KLASSEN VON SUBHARMONISCHEN UND VERWANDTEN FUNKTIONEN

voN

ALEXANDER DINGHAS

H E L S I N K I 1963

S U OMALA INEN TIEDEAKATEMIA 
Am 14. September 1962 vorgelegt von P. J. Myrberg und Olli Lehto 


\title{
Über das Anwachsen einiger Klassen von subharmonischen und verwandten Funktionen
}

\author{
Herrn Akitsugu Kawaguchi zum 65. Geburtstag
}

1. Einleitung. Die Funktion $u(z)(z=x+i y ; x, y$ reell $)$ sei in $H: x>0$ und habe dort die Eigenschaften:

1) $u$ ist in $H$ subharmonisch.

2) Es ist $u(z) \geqq 0$ in $H$.

3) Für jeden endlichen Randpunkt $\zeta$ von $H$ gilt

$$
\lim _{z \rightarrow \zeta} u(z)=0 .
$$

Genügt eine in $H$ subharmonische Funktion der Bedingung

$$
\varlimsup_{z \rightarrow \zeta} u(z) \leqq 0,
$$

so wird sie von der Klasse $L_{0}$ genannt. Offenbar bildet die Gesamtheit $L$ aller Funktionen, die den Bedingungen 1) -3) genügen, eine Teilklasse von $L_{\mathbf{0}}$. Sie soll im folgenden durch $L$ bezeichnet werden. Ist $u \in L_{\mathbf{0}}$ und $\stackrel{+}{u}(z)=\operatorname{Max}\{0, u(z)\}$, so ist stets $\stackrel{+}{u} \in L$.

Neben den Klassen $L_{0}$ und $L$ betrachten wir im folgenden die Funktionenklassen $J$ bzw. $J_{0}$ von superharmonischen Funktionen mit den Eigenschaften:

$$
\begin{aligned}
v(z)>0 & (v \in J), \\
\varliminf_{z \rightarrow \zeta} v(z) \geqq 0 &
\end{aligned}
$$$$
\text { b) }
$$

Für die Funktionen der Klasse $L$ und für Funktionen der Klassen $L, L_{0}, J$ und $J_{0}$ gelten nun eine Reihe von Sätzen, deren wichtigster Teil den Problemkreis des klassischen Phragmén-Lindelöfschen Prinzips und des Schwarz-Carathéodory-Juliaschen Lemmas umspannt. Hierzu dürfte noch eine Reihe von Sätzen (deren älteste wohl auf Ahlfors und Carleman zurückgehen) gezählt werden, welche auf die Konvexität von bestimmten Mittelwerten (und somit deren Monotonie) hinweisen. 
In der vorliegenden Arbeit sollen zwei allgemeine Konvexitätssätze für die Funktionen der beiden Klassen $L$ bzw. $J$ bewiesen werden, die nicht nur die vorhin genannten Sätze umfassen und vertiefen, sondern noch durch Grenzübergang gestatten, über das Anwachsen der Funktionen von $L_{0}$ und $J_{0}$ allgemeine Aussagen zu machen. Einige Anwendungen auf die (konformen) Minimalabbildungen sowie auf die subelliptischen Funktionen findet der Leser in 8 und 10.

2. Mittelbildungen. Formulierung der Hauptsätze. Bekanntlich wird die in einem (beschränkten) Gebiet $G$ der komplexen Ebene definierte Funktion $u(z)$ als subharmonisch bezeichnet, wenn sie folgenden Bedingungen genügt:

1. $-\infty \leqq u(z)<+\infty, \quad u(z) \neq-\infty$.

2. $u(z)$ ist in $G$ nach oben halbstetig.

3. Es sei $G_{1}$ ein (beliebiges) Teilgebiet von $G$ (mit $\bar{G}_{1} \subset G$ ), dessen Rand $\Gamma$ aus endlich vielen Jordanschen Kurven besteht, und $h(z)$ die harmonische Funktion in $G$ mit den Randwerten $u(\zeta)(\zeta \in \Gamma)$.

Dann ist

$$
u(z) \leqq h(z) \quad\left(z \in G_{1}\right) .
$$

Entsprechend heisst $v(z)$ superharmonisch in $G$, wenn folgende Bedingungen erfüllt sind:

1. $-\infty<v(z) \leqq+\infty, \quad v(z) \neq \infty$.

2. $v(z)$ ist nach unten halbstetig.

3. Es gilt

$$
v(z) \geqq h(z) \quad\left(z \in G_{1}\right) .
$$

Ist $u(z)$ subharmonisch in $G$, so ist offenbar - $u(z)$ dort superharmonisch.

Man setze jetzt $z=r e^{i \vartheta}(0<r<+\infty,|\vartheta|<\pi / 2)$ und für jedes $\alpha \in[2,+\infty)$

$$
L_{\alpha}(r, u)=\left\{\int_{-\frac{\pi}{2}}^{\frac{\pi}{2}}\left(\frac{u}{r \cos \vartheta}\right)^{\alpha} \cos ^{2} \vartheta d \vartheta\right\}^{\frac{1}{\alpha}} \quad(u \in L)
$$

und für $\alpha \in[1,+\infty)$

$$
J_{\alpha}(r, v)=\left\{\int_{-\frac{\pi}{2}}^{\frac{\pi}{2}}\left(\frac{v}{r \cos \vartheta}\right)^{-\alpha} \cos ^{2} \vartheta d \vartheta\right\}^{-\frac{1}{\alpha}} \quad(v \in J)
$$


mit $u=u\left(r e^{i \vartheta}\right), v=v\left(r e^{i \vartheta}\right)$. Hierbei sind beide Integrale rechts im Lebesgueschen Sinne zu nehmen und somit (nach klassischen Sätzen der Funktionentheorie und der Integrationstheorie) endlich. Für die Mittelwerte $L_{\alpha}(r, u)$ bzw. $J_{\alpha}(r, v)$ gelten nun die Sätze:

Satz 1. Es bedeute $I$ das Intervall $0<r<+\infty$. Dann ist jedes $L_{a}(r, u)(u \in L)$ in $I$ nicht abnehmend und $r^{2} L_{\alpha}(r, u)$ konvex in $r^{2}$.

Satz 2. Die Funktionen $J_{a}(r, v)(v \in J)$ sind in $I$ nicht zunehmend und $r^{2} J_{a}(r, v)$ konkav in $r^{2}$.

Betrachtet man eine Funktion, die in $H$ subharmonisch ist und dort der Lindelöfschen Bedingung (1.2) genügt, so ist für jedes $r \in I$ der Ausdruck

$$
L(r, u)=\sup \left\{\frac{u\left(r e^{i \vartheta}\right)}{r \cos \vartheta}|| \vartheta \mid<\frac{\pi}{2}\right\} \quad\left(u \in L_{0}\right)
$$

endlich. Setzt man entsprechend für eine in $H$ superharmonische Funktion $v(z)$, die der (Juliaschen) Bedingung b) genügt,

$$
J(r, v)=\inf \left\{\frac{v\left(r e^{i \vartheta}\right)}{r \cos \vartheta}|| \vartheta \mid<\frac{\pi}{2}\right\} \quad\left(v \in J_{0}\right),
$$

so kann man aus den Sätzen 1 und 2 folgende Sätze beweisen.

Satz 3. Die Funktion $L(r, u)$ ist in $I$ nicht abnehmend und $r^{2} L(r, u)$ konvex in $r^{2}$.

Satz 4. Die Funktion $J(r, v)$ ist in $I$ nicht zunehmend und $r^{2} J(r, v)$ konkav in $r^{2}$.

Die Sätze 3 und 4 (sowie auch 1 und 2) lassen sich leicht auf $M$ Abbildungen übertragen. Unter einer solchen Abbildung soll folgendes verstanden werden: Es seien $w_{1}(z), w_{2}(z), w_{3}(z)$ drei in einem Gebiet $G$ der komplexen $z$-Ebene definierte holomorphe Funktionen mit der Eigenschaft

$$
w_{1}^{\prime}(z)^{2}+w_{2}^{\prime}(z)^{2}+w_{3}^{\prime}(z)^{2} \equiv 0 \quad(z \in G) .
$$

Man setze

$$
u_{k}(z)=h_{k}(z)+i h_{k}^{*}(z) \quad(k=1,2,3)
$$

mit reellen $h_{k}, h_{k}^{*}$ und betrachte in $E^{3}$ die Fläche

$$
F=\left\{\mathfrak{r} \mid \mathfrak{r}=\left(h_{1}(z), h_{2}(z), h_{3}(z)\right), z \in G\right\} .
$$

Dann soll die Abbildung $F: z \rightarrow \mathfrak{r}$ (mit Rücksicht auf den Zusammenhang mit der Theorie der Minimalflächen) eine $M$-Abbildung heissen. Man setze jetzt

$$
H(z)=\log \sqrt{h_{1}(z)^{2}+h_{2}(z)^{2}+h_{3}(z)^{2}}
$$

und nehme an, $G$ falle mit $H$ zusammen. Dann gelten die Sätze: 
Satz 5. Es sei $H \rightarrow F$ eine $M$-Abbildung derart, dass $H(z)$ der (Lindelöfschen.) Bedingung

$$
\varlimsup_{z \rightarrow ;} H(z) \leqq 0
$$

genügt. Man setze

$$
H(r, \mathfrak{r})=\sup \left\{\frac{H\left(r e^{i \vartheta}\right)}{r \cos \vartheta}|| \vartheta \mid<\frac{\pi}{2}\right\} .
$$

Dann ist $H(r, \mathfrak{r})$ in $I$ nicht abnehmend und $r^{2} H(r, \mathfrak{r})$ konvex in $r^{2}$. Ist

$$
\varlimsup_{r \rightarrow+\infty} H(r, \mathfrak{r})>0,
$$

so gilt für die Ausdrücke

$$
H_{\alpha}(r, \mathfrak{r})=\left\{\int_{-\frac{\pi}{2}}^{+\frac{\pi}{2}}\left(\frac{+}{r} \frac{+\left(r e^{i \vartheta}\right)}{\cos \vartheta}\right)^{\alpha} \cos ^{2} \vartheta d \vartheta\right\}^{\frac{1}{\alpha}}
$$

$(\alpha \in[2,+\infty))$ der (nicht triviale) Satz 1.

3. Vorbereitende Hilfsbetrachtungen. Dem Beweis der Konvexitätssätze von 2 sollen folgende Hilfssätze vorangeschickt werden:

Hilfssatz 1. Die reelle Funktion $y(x)$ (kurz $y)$ sei im Intervall

$$
I_{0}: a \leqq x \leqq b \quad\left(-\frac{\pi}{2} \leqq a<b \leqq \frac{\pi}{2}\right)
$$

zweimal stetig differenzierbar und in|

$$
I_{0}^{*}: a<x<b
$$

positiv. Ist dann $y(a)=y(b)=0$, so ist für jedes $\alpha \in[0,+\infty)$

$$
J_{\alpha}(y)=\int_{I_{0}}\left(\frac{y}{\cos x}\right)^{\alpha-1} y\left(y+y^{\prime \prime}\right) d x \leqq 0 .
$$

Das Gleichheitszeichen tritt hier dann und nur dann ein, wenn $y=C \cos x$ mit einem konstanten $C$ ist.

Hilfssatz 2. Die reelle Funktion $y(x)$ sei im Intervall

$$
I_{0}:-\frac{\pi}{2} \leqq x \leqq \frac{\pi}{2}
$$


zweimal stetig differenzierbar. Gilt dann $y(x)>0$ in $I_{0}$, so ist für jedes $\alpha \in(-\infty, 0]$

$$
J_{\alpha}(y)=\int_{I_{0}}\left(\frac{y}{\cos x}\right)^{\alpha-1} y\left(y+y^{\prime \prime}\right) d x \geqq 0 .
$$

Auch hier tritt das Gleichheitszeichen dann und nur dann ein, wenn $y=$ $C \cos x$ mit einem konstanten $C$ ist.

Zum Beweis der Hilfssätze 1 und 2 setze man $y=\sigma \cos x(\sigma=\sigma(x))$ und bezeichne den Integranden links in (3.1) durch $D_{\alpha}$. Dann wird wegen

$$
\begin{gathered}
y+y^{\prime \prime}=-2 \sigma^{\prime} \sin x+\sigma^{\prime \prime} \cos x \\
D_{\alpha}=\sigma^{\alpha}\left(-2 \sigma^{\prime} \sin x \cos x+\sigma^{\prime \prime} \cos ^{2} x\right)=\sigma^{\alpha}\left(\sigma^{\prime} \cos ^{2} x\right)^{\prime},
\end{gathered}
$$

und somit zunächst für jedes Paar $\left(x_{1}, x_{2}\right)$ mit $a<x_{1}<x_{2}<b$

$$
\int_{x_{1}}^{x_{2}}\left(\frac{y}{\cos x}\right)^{\alpha-1} y\left(y+y^{\prime \prime}\right) d x=\left.\sigma^{\alpha}\left(\sigma^{\prime} \cos ^{2} x\right)\right|_{x_{1}} ^{x_{2}}-\alpha \int_{x_{1}}^{x_{2}} \sigma^{\alpha-1} \sigma^{\prime 2} \cos ^{2} x d x .
$$

Es sei jetzt

$$
q=q(x)=\sigma(x)^{\alpha} \sigma^{\prime}(x) \cos ^{2} x
$$

gesetzt. Dann wird im Falle des Hilfssatzes 1 wegen $y^{\prime}(a) \geqq 0, y^{\prime}(b) \leqq 0$

und mithin auch

$$
\left.\lim _{\substack{x_{1} \rightarrow a \\ x_{2} \rightarrow b}} q(x)\right|_{x_{1}} ^{x_{2}} \leqq 0
$$

$$
\int_{a}^{b}\left(\frac{y}{\cos x}\right)^{a-1} y\left(y+y^{\prime \prime}\right) d x \leqq-\alpha \int_{a+}^{b-} \sigma^{\alpha-1} \sigma^{2} \cos ^{2} x d x .
$$

Liegt der Fall des Hilfssatzes 2 vor, so wird wegen

$$
\left.\lim _{\substack{x_{1} \rightarrow-\pi / 2 \\ x_{2} \rightarrow+\pi / 2}} q(x)\right|_{x_{1}} ^{x_{3}}=0
$$

$$
\int_{-\frac{\pi}{2}}^{\frac{\pi}{2}}\left(\frac{y}{\cos x}\right)^{\alpha-1} y\left(y+y^{\prime \prime}\right) d x=-\alpha \int_{-\frac{\pi}{2}}^{\frac{\pi}{2}} \sigma^{\alpha-1} \sigma^{\prime 2} \cos ^{2} x d x \geqq 0 .
$$

Soll in (3.1) bzw. (3.3) das Gleichheitszeichen gelten, so muss $\sigma$ konstant sein und somit $y=C \cos x$. Im Falle des Hilfssatzes 1 muss dann $a=$ $-\pi / 2, \quad b=\pi / 2$ sein. 
Zur Geschichte der Hilfssätze 1 und 2 sei hier kurz folgendes bemerkt: Der Fall $\alpha=0$ geht auf Ahlfors [1] (man vgl. auch Dinghas [4], [5]) zurück. Der Fall $\alpha=1$ bildet den Inhalt eines klassischen Lemmas der Variationsrechnung (man vgl. etwa: Blaschke [3]). Schliesslich wurden die Fälle $\alpha>2$ erstmalig von Dinghas [6] behandelt. Der Hilfssatz 2 ist neu.

4. Beweis des Satzes 1. Es sei $R>0$ fest, sonst willkürlich. Dann gibt es zu jedem $\varepsilon>0$ ein positives $\eta=\eta(\varepsilon, R)$ mit $\lim \eta(\varepsilon, R)=0$ derart, dass für alle $y,|y| \leqq R, u_{\varepsilon}(z)=u(z+\varepsilon)-\eta \leqq 0^{\varepsilon \rightarrow 0}$ gilt. Dann lässt sich $\stackrel{+}{u}_{\varepsilon}(z)$ in

$$
\bar{H}_{2 R}=\{z|| z \mid \leqq 2 R, \operatorname{Re} z \geqq 0\}
$$

als Grenzfunktion einer nicht zunehmenden Folge $\left(\tilde{u}_{n}(z)\right) \quad(n=1,2, \ldots)$ von zweimal stetig differenzierbaren Funktionen $\tilde{u}_{n}(z)$ darstellen, die alle in $L$ liegen $^{1}$ ). Somit kann man beim Beweis des Satzes 1 zunächst ohne weiteres annehmen, dass $u(z)$ eine in $\bar{H}_{2 R}$ nicht negative, zweimal stetig differenzierbare Funktion ist, die noch der Bedingung $u(i y)=0$ für alle $y$ mit $|y| \leqq R$ genügt. Man setze zur Abkürzung

$$
\psi=\psi(z)=\frac{u(z)}{\cos \vartheta} \quad\left(z \in \bar{H}_{R}\right)
$$

und bilde

$$
\mu=\mu(r)=\int_{-\frac{\pi}{2}}^{\frac{\pi}{2}} \psi\left(r e^{i \vartheta}\right)^{2 k} \cos ^{2} \vartheta d \vartheta \quad(k \geqq \mathrm{~J}) .
$$

Dann wird

$$
\mu^{\prime}=2 k \int_{-\frac{\pi}{2}}^{\frac{\pi}{2}} \psi^{2 k-1} u_{r} \cos \vartheta d \vartheta
$$

1) Man vgl. etwa: Tsuji [14], S. 44.

Der in Frage kommende Satz lautet folgendermassen:

Es sei $u(z)$ in $G$ subharmonisch und $\vec{G}_{1} \subset G$, und es bezeichne $A_{\varrho}^{(3)}(z, u)\left(z \in G_{1}\right)$ die Mittelung dritten Grades (danach ist $\pi \varrho^{2} A_{\varrho}{ }^{(1)}(z, u)=\int_{|\zeta-z| \leqq \varrho} u(\zeta) d \xi d \eta$ ) von $u(z)$ mit einem hinreichend kleinen @ (kleiner als die Entfernung von $G$ und $G_{1}$ ). Setzt man dann (bei festem $\varrho) ~ u_{\varrho}(z)=A_{\varrho}{ }^{(3)}(z, u)$, so ist $u_{\varrho}(z)$ 1) subharmonisch und zweimal stetig differenzierbar in $\left.G_{1}, 2\right)$ bei abnehmendem $\varrho$ nicht zunehmend und 3 ) konvergiert mit $\varrho \rightarrow 0$ gegen $u(z)$.

In dem uns interessierenden Fall kann leicht gezeigt werden, dass jedes $\tilde{u}_{n}(z)=u_{1 / n}(z)$ (für alle hinreichend grosse $n$ ) auf der Strecke $x=0,|y| \leqq R$ (man mache die Abschätzungen etwa in $\bar{H}_{2 R}$ ) verschwinden muss. 
und

$$
\mu^{\prime \prime} \geqq 2 k(2 k-1) \int_{-\frac{\pi}{2}}^{\frac{\pi}{2}} \psi^{2 k-2} u_{r}^{2} d \vartheta+2 k \int_{-\frac{\pi}{2}}^{\frac{\pi}{2}} \psi^{2 k-1} u_{r r} \cos \vartheta d \vartheta
$$

Nun folgt aus (4.4) (durch Anwendung der Schwarzschen Ungleichung)

$$
\mu^{\prime 2} \leqq 4 k^{2} \mu \int_{-\frac{\pi}{2}}^{\frac{\pi}{2}} \psi^{2 k-2} u_{r}^{2} d \vartheta
$$

Andererseits ist wegen

$$
\begin{gathered}
u_{r r}+\frac{1}{r} u_{r}+\frac{1}{r^{2}} u_{\vartheta \vartheta} \geqq 0 \\
2 k \int_{-\frac{\pi}{2}}^{\frac{\pi}{2}} \psi^{2 k-1} u_{r r} \cos \vartheta d \vartheta \geqq-\frac{\mu^{\prime}}{r}-2 k \frac{A}{r^{2}}
\end{gathered}
$$

mit

$$
A=\int_{-\frac{\pi}{2}}^{\frac{\pi}{2}} \psi^{2 k-2} u u_{\vartheta \vartheta} d \vartheta
$$

Jetzt wende man den Hilfssatz 1 auf alle (endlich vielen) Teilintervalle von $-\pi / 2 \leqq \vartheta \leqq \pi / 2$ an, auf denen $u$ positiv ist. Dann erhält man durch Summation $A \leqq-\mu$ und mithin die Differentialungleichung

$$
\mu\left(\mu^{\prime \prime}+\frac{\mu^{\prime}}{r}\right) \geqq \frac{2 k-1}{2 k}-\mu^{\prime 2}+\frac{2 k}{r^{2}} \mu^{2} .
$$

Es bedeute jetzt $R_{0}$ die untere Grenze aller $r$ für die $\mu(r)>0$ ist. Man nehme $R>R_{0}(R$ endlich) und betrachte das Ringgebiet

$$
T: R_{0}<r_{1} \leqq|z| \leqq r_{2}<R \quad\left(r_{1}<r_{2}\right)
$$

Dann folgt aus (4.8)

$$
\left.\frac{\mu^{\prime \prime}}{\mu}+\frac{\mu^{\prime}}{r \mu}-\frac{2 k-1}{2} \frac{\mu^{\prime}}{\mu}\right)^{2}-\frac{2 k}{r^{2}} \geqq 0
$$


und somit, wenn man

$$
\mu=\left(\frac{y}{r}\right)^{2 k}
$$

setzt, wegen

$$
\frac{\mu^{\prime}}{\mu}=2 k\left(\frac{y^{\prime}}{y}-\frac{1}{r}\right)
$$

und

$$
\begin{gathered}
\frac{\mu^{\prime \prime}}{\mu}-\left(\frac{\mu^{\prime}}{\mu}\right)^{2}=2 k\left\{\frac{y^{\prime \prime}}{y}-\left(\frac{y^{\prime}}{y}\right)^{2}+\frac{1}{r^{2}}\right\}, \\
y^{\prime \prime}-\frac{y^{\prime}}{r}=2 \frac{d}{d r}\left(\frac{d y}{d r^{2}}\right) \geqq 0 .
\end{gathered}
$$

Letztere Ungleichung hat die Konvexität von $y(r)$ in Bezug auf $r^{2}$ zur Folge.

Es gilt also für jedes $r \in\left[r_{1}, r_{2}\right]$

$$
r \mu(r) \leqq r_{1} \mu\left(r_{1}\right) \frac{r_{2}^{2}-r^{2}}{r_{2}^{2}-r_{1}^{2}}+r_{2} \mu\left(r_{2}\right) \frac{r^{2}-r_{1}^{2}}{r_{2}^{2}-r_{1}^{2}} .
$$

Diese Ungleichung, angewandt auf jede Funktion $\tilde{u}_{n}(z)$ der Approximationsfolge $\left(\tilde{u}_{n}(z)\right)$, liefert nun durch Grenzübergang $n \rightarrow+\infty$ die Konvexität von $r^{2} L_{a}\left(r, \stackrel{+}{u}_{\varepsilon}\right)$ als Funktion von $r^{2}$. Der endgültige Beweis des Satzes 1 folgt daraus, indem man $\varepsilon \rightarrow 0$ konvergieren lässt. Da nun noch bei gegebenen $r_{1}, r_{2} \in(0,+\infty)$ sich stets ein $R>\operatorname{Max}\left(r_{1}, r_{2}\right)$ für die Bildung von $\stackrel{+}{u}_{\varepsilon}(z)$ wählen lässt, so gilt allgemein

$$
r^{2} L_{\alpha}(r, u) \leqq r_{1}^{2} L_{a}\left(r_{1}, u\right) \frac{r_{2}^{2}-r^{2}}{r_{2}^{2}-r_{1}^{2}}+r_{2}^{2} L_{\alpha}\left(r_{2}, u\right) \frac{r^{2}-r_{1}^{2}}{r_{2}^{2}-r_{1}^{2}} .
$$

Der Satz 3 lässt sich aus (4.14) durch Grenzübergang $\alpha \rightarrow+\infty$ leicht ableiten. Mit Rücksicht jedoch darauf, dass man für diesen Spezialfall einen eleganten und kurzen Beweis geben kann, sollen die diesbezüglichen Überlegungen dem Leser überlassen werden. Ebenso kann der Leser zeigen (am einfachsten durch Anwendung des Gaussschen Satzes), dass die Ungleichungen (4.4), (4.5) (allgemein mit den unteren Derivierten anstelle $\mu^{\prime \prime}$ ) und (4.10) auch dann gelten, wenn $2 k=1$ ist. Das hat zur Folge, dass $r \mu$ (gebildet für $2 k=1$ ) konvex in $r^{2}$ ist. Man vgl. auch Ahlfors [1] und Dinghas [5].

5. Beweis des Satzes 2. Beim Beweis des Satzes 2 empfiehlt es sich, zunächst von der Funktion $v(z+\varepsilon)+\varepsilon$ auszugehen und die (bei gegebenem 
$\varepsilon>0$ und $R>0$ existierende) Approximationsfolge $\left(\tilde{v}_{n}(z)\right)$ heranzuziehen. Dabei kann (sofern $v(z)$ nicht in $H$ identisch verschwindet) angenommen werden, dass jede der (zweimal stetig differenzierbaren) superharmonischen Funktionen $v_{n}(z)$ in $H_{2 R}$ positiv ist. Man schreibe nun $v(z)$ für ein $\tilde{v}_{n}(z)$ und setze ähnlich wie vorhin

$$
\sigma=\sigma(r)=\int_{-\frac{\pi}{2}}^{\frac{\pi}{2}} \psi\left(r e^{i \vartheta}\right)^{2 k} \cos ^{2} \vartheta d \vartheta \quad\left(k \geqq \frac{1}{2}\right)
$$

mit

$$
\psi(z)=\frac{\cos \vartheta}{v(z)} \quad\left(z \in \bar{H}_{R}, z=r e^{i \vartheta}\right) .
$$

Dann findet man

$$
\sigma^{\prime}=-2 k \int_{-\frac{\pi}{2}}^{\frac{\pi}{2}} \psi^{2 k+1} v_{r} \cos \vartheta d \vartheta
$$

und

$$
\sigma^{\prime \prime}=2 k(2 k+1) \int_{-\frac{\pi}{2}}^{\frac{\pi}{2}} \psi^{2 k+2} v_{r}^{2} d \vartheta-2 k \int_{-\frac{\pi}{2}}^{\frac{\pi}{2}} \psi^{2 k+1} v_{r r} \cos \vartheta d \vartheta
$$

Nun ist

$$
\sigma^{2} \leqq 4 k^{2} \sigma \int_{-\frac{\pi}{2}}^{\frac{\pi}{2}} \psi^{2 k+2} v_{r}^{2} d \vartheta
$$

und

$$
v_{r r}+\frac{1}{r} v_{r}+\frac{1}{r^{2}} v_{\vartheta \vartheta} \leqq 0 .
$$

Das liefert (unter Heranziehung des Hilfssatzes 2) die Differentialungleichung

$$
\frac{\sigma^{\prime \prime}}{\sigma}+\frac{\sigma^{\prime}}{r \sigma} \geqq \frac{2 k+1}{2 k}\left(\frac{\sigma^{\prime}}{\sigma}\right)^{2}-\frac{2 k}{r^{2}} .
$$

Man setze jetzt, ähnlich wie in 3 ,

$$
\frac{1}{\sigma}=\left(\frac{y}{r}\right)^{2 k}
$$


also $-\log \sigma=2 k(\log y-\log r)$. Dann wird

$$
\frac{\sigma^{\prime}}{\sigma}=-2 k\left(\frac{y^{\prime}}{y}-\frac{1}{r}\right) \text {. }
$$

und

$$
\frac{\sigma^{\prime \prime}}{\sigma}-\left(\frac{\sigma^{\prime}}{\sigma}\right)^{2}=-2 k\left\{\frac{y^{\prime \prime}}{y}-\left(\frac{y^{\prime}}{y}\right)^{2}+\frac{1}{r^{2}}\right\} .
$$

Daraus folgt leicht

$$
-2 k\left(\frac{y^{\prime \prime}}{y}-\frac{y^{\prime}}{r y}\right) \geqq 0,
$$

d. h.

$$
r y^{\prime \prime}-y^{\prime}=2 r^{2} \frac{d}{d r}\left(\frac{d y}{d r^{2}}\right) \leqq 0 .
$$

Das beweist die Konkavität von $y$ als Funktion von $r^{2}$. Hat man nun die Ungleichung

$$
y(r) \geqq y\left(r_{1}\right) \frac{r_{2}^{2}-r^{2}}{r_{2}^{2}-r_{1}^{2}}+y\left(r_{2}\right) \frac{r^{2}-r_{1}^{2}}{r_{2}^{2}-r_{1}^{2}}
$$

für jedes Intervall $0<r_{1}<r<r_{2}<+\infty$ bewiesen, so liefern die Grenzübergänge $n \rightarrow+\infty, \varepsilon \rightarrow 0$ leicht die Ungleichung

$$
r^{2} J_{a}(r, v) \geqq r_{1}^{2} J_{a}\left(r_{1}, v\right) \frac{r_{2}^{2}-\cdots r^{2}}{r_{2}^{2}-r_{1}^{2}}+r_{2}^{2} J_{a}\left(r_{2}, v\right) \frac{r^{2}-r_{1}^{2}}{r_{2}^{2}-r_{1}^{2}}
$$

für die ursprünglich in 1 betrachtete Funktion $v(z)$. Dabei ist hier wieder $\left.\alpha \in[1,+\infty)^{2}\right)$.

6. Beweis der Sätze 3 und 4. Es bedeute $H_{1}$ den Halbring

$$
0<r_{1} \leqq|z| \leqq r_{2}, \quad \operatorname{Re} z \geqq 0 \quad\left(r_{1}<r_{2}<+\infty\right),
$$

$\Gamma_{1}, \Gamma_{2}$ die Halbperipherien $|z|=r_{1}(\operatorname{Re} z \geqq 0),|z|=r_{2}(\operatorname{Re} z \geqq 0)$ und $g(z, \zeta)$ (kurz $g$ ) die Greensche Funktion des offenen Kerns $H_{1}^{*}$ von $H_{1}$. Wir setzen $z=r e^{i \vartheta}\left(z \in H_{1}^{*}\right), \zeta=|\zeta| e^{i \vartheta}$, bezeichnen durch $\Gamma$ den gesamten Rand von $H_{1}$ und schreiben $\partial / \partial n$ für die Differentiation nach der inneren Normale von $\Gamma$ im Punkt $\zeta \in \Gamma$. Dann wird (mit Rücksicht darauf, dass die Realteile von $\zeta$ und $1 / \zeta$ auf $\Gamma \backslash \Gamma_{1} \cup \Gamma_{2}$ verschwinden)

$$
r \cos \vartheta=\frac{1}{2 \pi} \int_{I_{1} \cup \Gamma_{2}} \operatorname{Re} \zeta \frac{\partial g}{\partial n} d s
$$

2) Eine Beweisskizze der Sätze 1 und 2 habe ich zuerst in einem Anfang 1962 (man vgl. [7]) an der Technischen Hochschule Trondheim gehaltenen Vortrag gegeben. 
und

$$
\frac{\cos \vartheta}{r}=\frac{1}{2 \pi} \int_{I_{1} \cup \Gamma_{\mathbf{z}}} \operatorname{Re} \frac{1}{\zeta} \frac{\partial g}{\partial n} d s
$$

wobei $d s$ das Bogenelement von $\Gamma$ in $\zeta$ bedeutet.

Man definiere nun $\alpha_{1}, \alpha_{2}$ durch die Gleichungen

$$
\alpha_{1}=\frac{1}{2 \pi} \int_{\Gamma_{1}^{\prime}} \frac{\partial g}{\partial n} \cos \varphi d s
$$

und

$$
\alpha_{2}=\frac{1}{2 \pi} \int_{\Gamma_{2}} \frac{\partial g}{\partial n} \cos \varphi d s .
$$

Dann folgt aus (6.1) und (6.2)

$$
\alpha_{1}=\frac{r_{2}^{2}-r^{2}}{r_{2}^{2}-r_{1}^{2}} \frac{r_{1}}{r} \cos \vartheta
$$

und

$$
\alpha_{2}=\frac{r^{2}-r_{1}^{2}}{r_{2}^{2}-r_{1}^{2}} \frac{r_{2}}{r} \cos \vartheta .
$$

Es sei jetzt $u(z)$ in $H$ subharmonisch und genüge der Lindelöfschen Bedingung (1.1). Dann ist

$$
H(z)=\frac{1}{2 \pi} \int_{\Gamma_{1}} u(\zeta) \frac{\partial g}{\partial n} d s+\frac{1}{2 \pi} \int_{\Gamma_{\mathbf{z}}} u(\zeta) \frac{\partial g}{\partial n} d s
$$

(wobei $\Gamma_{1}, \Gamma_{2}$ positiv orientiert sind) eine harmonische Majorante von $u(z)$, sofern $z$ in $H_{1}^{*}$ liegt. Nun ist

$$
\frac{1}{2 \pi} \int_{I_{1}} u(\zeta) \frac{\partial g}{\partial n} d s \leqq L\left(r_{1}, u\right) r_{1} \alpha_{1}
$$

und

$$
\frac{1}{2 \pi} \int_{\Gamma_{2}} u(\zeta) \frac{\partial g}{\partial n} d s \leqq L\left(r_{2}, u\right) r_{2} \alpha_{2}
$$

also

$$
r \frac{u(z)}{\cos \vartheta} \leqq r_{1}^{2} L\left(r_{1}, u\right) \frac{r_{2}^{2}-r^{2}}{r_{2}^{2}-r_{1}^{2}}+r_{2}^{2} L\left(r_{2}, u\right) \frac{r^{2}-r_{1}^{2}}{r_{2}^{2}-r_{1}^{2}}
$$


Geht man hier links (bei festem $r, r_{1}<r<r_{2}$ ) zum Supremum über, so erhält man die Ungleichung

$$
r^{2} L(r, u) \leqq r_{1}^{2} L\left(r_{1}, u\right) \frac{r_{2}^{2}-r^{2}}{r_{2}^{2}-r_{1}^{2}}+r_{2}^{2} L\left(r_{2}, u\right) \frac{r^{2}-r_{1}^{2}}{r_{2}^{2}-r_{1}^{2}} .
$$

Will man die Konkavität von $r^{2} J(r, v)$, wobei $v(z)$ in $z \in H$ superharmonisch ist und der Bedingung b) genügt, beweisen, so muss man von der Ungleichung $v(z) \geqq H(z) \quad\left(z \in H_{1}^{*}\right)$ ausgehen und die rechte Seite nach unten durch den Ausdruck

$$
r_{1} \alpha_{1} J\left(r_{1}, v\right)+r_{2} \alpha_{2} J\left(r_{2}, v\right)
$$

abschätzen. Man erhält dann die Ungleichung

$$
r \frac{v\left(r e^{i \vartheta}\right)}{\cos \vartheta} \geqq r_{1}^{2} J\left(r_{1}, v\right) \frac{r_{2}^{2}-r^{2}}{r_{2}^{2}-r_{1}^{2}}+r_{2}^{2} J\left(r_{2}, v\right) \frac{r^{2}-r_{1}^{2}}{r_{2}^{2}-r_{1}^{2}}
$$

und somit auch die Abschätzung

$$
r^{2} J(r, v) \geqq r_{1}^{2} J\left(r_{1}, v\right) \frac{r_{2}^{2}-r^{2}}{r_{2}^{2}-r_{1}^{2}}+r_{2}^{2} J\left(r_{2}, v\right) \frac{r^{2}-r_{1}^{2}}{r_{2}^{2}-r_{1}^{2}}
$$

Lässt man in (6.8) und (6.9) $r_{1} \rightarrow 0$ konvergieren, so erhält man die Ungleichungen

$$
L(r, u) \leqq L\left(r_{2}, u\right)
$$

und

$$
J(r, v) \geqq J\left(r_{2}, v\right),
$$

welche die Monotonie von $L(r, u)$ und $J(r, v)$ zum Ausdruck bringen (man vgl. in diesem Zusammenhang die zusammenfassende Note [8]).

7. Nochmals die Sätze von Phragmén-Lindelöf und Julia. Die vorhin entwickelte kurze Methode zum Beweis der Sätze 3 und 4 kann durch eine einfachere ersetzt werden (in dem Sinne, dass der Begriff der Greenschen Funktion nicht benutzt wird), sofern es sich um die klassischen Sätze von Phragmén-Lindelöf und Julia handelt [4].

Es sei

$$
H(\zeta, z)=\operatorname{Re}\left\{\frac{\zeta+z}{\bar{\zeta}-z}-\overline{\bar{\zeta}} \frac{\overline{\bar{\zeta}}-z}{\bar{\zeta}}\right\}
$$

und es bedeute $H_{R}$ den Halbkreis

$$
|\zeta| \leqq R, \quad \operatorname{Re} \zeta>0 .
$$


Ist dann $u(z)$ subharmonisch in $H$ und genügt sie der Lindelöfschen Bedingung (1.2), so ist

$$
\Phi(z, u)=\frac{1}{2 \pi} \int_{-\frac{\pi}{2}}^{\frac{\pi}{2}} u(\zeta) H(\zeta, z) d \varphi
$$

$\left(\zeta=R e^{i \varphi}, \quad z=r e^{i \vartheta}, 0<r<R\right)$ eine harmonische Majorante für $u(z)$ in $H_{R}$. Ist dagegen $v(z)$ superharmonisch in $H$ und genügt sie dort der (Juliaschen) Bedingung b), so ist

$$
\Phi(z, v)=\frac{1}{2 \pi} \int_{-\frac{\pi}{2}}^{\frac{\pi}{2}} v(\zeta) H(\zeta, z) d \varphi
$$

eine harmonische Minorante von $v(z)$ in $H_{R}$.

Nun ist mit Rücksicht auf die Entwicklung

$$
H(\zeta, z)=4 \sum_{k=1}^{\infty}\left(\frac{r}{R}\right)^{k} \psi_{k}(\vartheta) \psi_{k}(\varphi)
$$

mit

$$
\psi_{k}(\alpha)= \begin{cases}\cos k \alpha & \text { für } k \text { ungerade } \\ \sin k \alpha & \text { für } k \text { gerade }\end{cases}
$$

$$
\frac{1}{2 \pi} \int_{-\frac{\pi}{2}}^{\frac{\pi}{2}} H(\zeta, z) \cos \varphi d \varphi=\frac{r}{R} \cos \vartheta
$$

und mithin wegen

$$
u(z) \leqq L(R, u) \cdot \frac{1}{2 \pi} \int_{-\frac{\pi}{2}}^{\frac{\pi}{2}} H(\zeta, z) \cos \varphi d \varphi
$$

und

$$
\begin{gathered}
v(z) \geqq J(R, v) \cdot \frac{1}{2 \pi} \int_{-\frac{\pi}{2}}^{\frac{\pi}{2}} H(\zeta, z) \cos \varphi d \varphi \\
\frac{u(z)}{r \cos \vartheta} \leqq L(R, u)
\end{gathered}
$$


und

$$
\frac{v(z)}{r \cos \vartheta} \geqq J(R, v) \quad\left(z=r e^{i \vartheta}\right) .
$$

Die Ungleichungen (7.6) sowie (7.7) haben die Monotonie von $L(r, u)$ bzw. $J(r, v)$ zur Folge und gestatten eine einheitliche Behandlung der Sätze von Phragmén-Lindelöf und Julia.

Letzterer Satz nimmt z. B. folgende Form an: Es sei $v(z)$ in $H$ superharmonisch und dort $\geqq 0$. Ist dann $\lim J(r, v)=c>0$, so gilt dort

$$
v(z) \geqq c x \quad(z=x+i y) .
$$

Der Satz 1, angewandt auf die Funktion $u(z)=\log |w(z)|$, wobei $w(z)$ in $H$ holomorph ist und dort der Lindelöfschen Bedingung

$$
\varlimsup_{z \rightarrow i \eta}|w(z)| \leqq 1 \quad(-\infty<\eta<+\infty)
$$

genügt, führt zu folgender Fassung des klassischen Phragmén-Lindelöfschen Prinzips:

Genügt die in $H$ holomorphe Funktion $w(z)$ der Bedingung (7.9), so existiert stets der Grenzwert

$$
\lim _{r \rightarrow+\infty} L(r, \log |w|)=\alpha .
$$

Ist hier $\alpha \leqq 0$, so muss wegen (7.6) in $H$

$$
|w(z)| \leqq e^{\alpha x} \quad(z=x+i y)
$$

gelten und mithin $|w(z)| \leqq 1$ sein. Ist $\alpha>0$, so gibt es mindestens einen Punkt $z_{0} \in H$ mit $\left|w\left(z_{0}\right)\right|>1$. Daraus folgt leicht aus der Ungleichung

$$
\log \left|w\left(z_{0}\right)\right| \leqq \Phi\left(z_{0}, \log |w|\right)
$$

wegen

$$
\Phi\left(z_{0}, \log |w|\right) \leqq \frac{1}{2 \pi} \int_{-\frac{\pi}{2}}^{\frac{\pi}{2}} \log |w(\zeta)| \cdot H(\zeta, z) d \varphi
$$

das Phragmén-Lindelöfsche Prinzip in der klassischen Fassung von F. und R. Nevanlinna [13].

Ich schliesse diese Nummer mit der Bemerkung, dass Ahlfors, M. Heins und Hayman noch die tieferliegende Tatsache bewiesen haben, dass sowohl im Lindelöfschen, wie auch im Juliaschen Falle (beide Fälle gehen durch Betrachtung der entgegengesetzten Funktion ineinander über) der radiale 
Grenzwert von $u\left(r e^{i \vartheta}\right) / r \cos \vartheta$ bzw. $v\left(r e^{i \vartheta}\right) / r \cos \vartheta$ bei festem $\vartheta,|\vartheta|<\pi / 2$, stets existiert, sofern $r$ ausserhalb einer $r$-Menge von endlicher logarithmischer Gesamtlänge gegen $+\infty$ konvergiert. Eine ausführliche Darstellung dieser Zusammenhänge findet der Leser in der Arbeit [9] von W. K. Hayman.

8. Das Phragmén-Lindelöfsche Prinzip für $M$-Abbildungen. Ist $\mathfrak{x}$ eine Minimalabbildung im Sinne der Entwicklungen von 2 und

$$
\exp H(z)=|\mathfrak{r}(z)|=\sqrt{h_{1}(z)^{2}+h_{2}(z)^{2}+h_{3}(z)^{2}},
$$

so ist $\log |\mathfrak{r}(z)|$ in $H$ subharmonisch. Das ist der Inhalt eines Satzes, den E. F. Beckenbach und T. Radó [2] im Jahre 1933 bewiesen haben. Damit der Leser diese Arbeit nicht zu lesen braucht, gebe ich kurz den Beweis dieses Satzes, für den uns interessierenden Spezialfall, mit der Bemerkung wieder, dass die diesbezüglichen Überlegungen auch dann erhalten bleiben, wenn anstelle $H$ ein beliebiges Gebiet genommen wird.

Man schreibe $u, v, w$ für $h_{1}, h_{2}, h_{3}$ und beachte, dass mit Rücksicht auf die Identität $w_{1}^{\prime 2}+w_{2}^{\prime 2}+w_{3}^{\prime 2} \equiv 0$ die Gleichungen

$$
u_{x}^{2}+v_{x}^{2}+w_{x}^{2}=u_{y}^{2}+v_{y}^{2}+w_{y}^{2}
$$

und

$$
u_{x} u_{y}+v_{x} v_{y}+w_{x} w_{y}=0
$$

gelten müssen. Es bezeichnen nun $\left|\mathfrak{r}_{x}\right|$ bzw. $\left|\mathfrak{r}_{\boldsymbol{y}}\right|$ die Längen der Vektoren mit den Koordinaten $u_{x}, v_{x}, w_{x}$ bzw. $u_{y}, v_{y}, w_{y}$. Dann ist

$$
\begin{gathered}
\Delta H=\frac{\partial^{2} H}{\partial x^{2}}+\frac{\partial^{2} H}{\partial y^{2}}=H_{x x}+H_{y y} \\
=\frac{\left|\mathfrak{r}_{x}\right|^{2}+\left|\mathfrak{r}_{y}\right|^{2}-2\left(\left|\mathfrak{r}_{x}\right|^{2} \cos ^{2} \alpha+\left|\mathfrak{r}_{y}\right|^{2} \cos ^{2} \beta\right)}{|\mathfrak{r}|^{2}},
\end{gathered}
$$

wobei $\alpha, \beta$ die Winkel von $\mathfrak{r}$ mit $\mathfrak{r}_{x}$ bzw. $\mathfrak{r}_{y}$ sind. Daraus folgt zunächst

$$
\Delta H=\frac{\left|\mathfrak{r}_{x}\right|^{2}+\left|\mathfrak{r}_{y}\right|^{2}}{|\mathfrak{r}|^{2}}\left(1-\cos ^{2} \alpha-\cos ^{2} \beta\right) .
$$

Dass nun die Klammer rechts nicht negativ ist, zeigt man so: Es sei $l$ die Projektion des Einheitsvektors $\left(\frac{u}{|\mathfrak{x}|}, \frac{v}{|\mathfrak{x}|}, \frac{w}{|\mathfrak{x}|}\right)$ auf die durch $\mathfrak{r}_{\boldsymbol{x}}, \mathfrak{r}_{\boldsymbol{y}}$ definierte (und durch $\mathfrak{r}$ gehende) Ebene $\Pi$. Dann ist $\cos ^{2} \alpha+\cos ^{2} \beta=$ $l^{2} \leqq 1$ und somit $1-\cos ^{2} \alpha-\cos ^{2} \beta \geqq 0$. Daraus folgt $\Delta H \geqq 0$ in allen Punkten $z$ mit $\mathfrak{r}(z) \neq 0$. Da nun noch in allen (isolierten) Punkten $z$ mit $\mathfrak{r}(z)=0 \quad H(z)=-\infty$ ist, so ist $H(z)$ subharmonisch in $H$. Soll 
$H(z)$ in $H$ harmonisch sein (also $\Delta H=0$ in jedem Punkt mit $\mathfrak{r}(z) \neq 0$, so muss $F$ bei der Abbildung $\mathfrak{x}: H \rightarrow F$ in einer Ebene liegen, die durch den Nullpunkt geht. Die Eigenschaft von $H(z)=\log |\mathfrak{r}(z)|$ in $H$ subharmonisch zu sein, führt zu folgender Übertragung des klassischen PhragménLindelöfschen Prinzips auf $M$-Abbildungen:

Satz 6. Es bedeute $\mathfrak{x}: H \rightarrow F$ eine $M$-Abbildung von $H$, für die die (Lindelöfsche) Bedingung

$$
\varlimsup_{z \rightarrow \text { in }}|\mathfrak{r}(z)| \leqq 1 \quad(-\infty<\eta<+\infty)
$$

gilt. Dann ist $|\mathfrak{r}(z)|$ entweder beschränkt in $H$ oder es gilt

$$
\lim _{r \rightarrow+\infty} \frac{M(r, \log |\mathfrak{x}|)}{r}>0 .
$$

Dabei ist

$$
M(r, \log |\mathfrak{r}|)=\sup \left\{\log \left|\mathfrak{r}\left(r e^{i \vartheta}\right)\right||\vartheta|<\frac{\pi}{2}\right\}
$$

Dieser Satz ist eine unmittelbare Folgerung des Satzes 5, d.h. der Monotonie von

$$
L(r, \mathfrak{r})=\sup \left\{\frac{\log \left|\mathfrak{r}\left(r e^{i \vartheta}\right)\right|}{r \cos \vartheta}|| \vartheta \mid<\frac{\pi}{2}\right\},
$$

die wieder eine Folgerung des Satzes 3 ist. Wir gehen darauf nicht ein.

Zum Schluss dürfte noch über das Eintreten des Gleichheitszeichens folgendes hinzugefügt werden: Ist $w_{1}(z)=u_{1}+i v_{1}=e^{\alpha z+\beta / z}$ mit reellen $\alpha, \beta(\beta \leqq 0)$, so ist $L\left(r, u_{1}\right)=\alpha+\beta / r^{2}$ und mithin $r^{2} L\left(r, u_{1}\right)=\alpha r^{2}+\beta$. Somit ist

$$
u_{1}=\left(\alpha r+\frac{\beta}{r}\right) \cos \vartheta
$$

Extremalfunktion für die Sätze 1 und 3. Entsprechend ist

$$
u_{2}=\left(\alpha r+\frac{\beta}{r}\right) \cos \vartheta \quad(\beta \geqq 0)
$$

eine Extremalfunktion für die Sätze 2 und 4. Fragt man nach Extremalfunktionen im Satz 5, so stellt man leicht fest, dass die holomorphen Funktionen $w_{k}=a_{k} e^{z}(k=1,2,3)$ mit $a_{1}^{2}+a_{2}^{2}+a_{3}^{2}=0$ und $\left|a_{1}\right|^{2}+\left|a_{2}\right|^{2}+$ $\left|a_{3}\right|^{2}=2$ eine extremale $M$-Abbildung liefern.

9. Der räumliche Fall. In dieser Nummer soll noch kurz die Übertragung der Sätze 1-4 auf den $n$-dimensionalen euklidischen Raum $E^{n} \quad(n>2)$ 
angedeutet werden. Es sei $P\left(x_{1}, \ldots, x_{n}\right)$ ein Punkt von $E^{n}$ und $S$ die Einheitskugel

$$
\xi_{1}^{2}+\xi_{2}^{2}+\cdots+\xi_{n}^{2}=1
$$

Wir setzen

$$
\begin{aligned}
\xi_{1} & =\cos \vartheta_{1} \\
\xi_{2} & =\sin \vartheta_{1} \cos \vartheta_{2} \\
\cdot & \cdot \cdot \cdot \\
\xi_{n-1} & =\sin \vartheta_{1} \sin \vartheta_{2} \ldots \sin \vartheta_{n-2} \cos \vartheta_{n-1} \\
\xi_{n} & =\sin \vartheta_{1} \sin \vartheta_{2} \ldots \sin \vartheta_{n-2} \sin \vartheta_{n-1}
\end{aligned}
$$

mit $0 \leqq \vartheta_{1}, \ldots, \vartheta_{n-2} \leqq \pi, 0 \leqq \vartheta_{n-1} \leqq 2 \pi$, und erhalten

$$
d s^{2}=\sum_{k=1}^{n} d \xi_{k}^{2}=\sum_{k=1}^{n-1} g_{k} d \vartheta_{k}^{2}
$$

mit $g_{k}=g_{k}\left(\vartheta_{1}, \ldots, \vartheta_{k-1}\right)$. Ferner setzen wir für zwei auf $S$ differenzierbare Funktionen $u, v^{3}$ )

$$
(\nabla u, \nabla v)=\sum_{k=1}^{n-1} \frac{1}{g_{k}} \frac{\partial u}{\partial \vartheta_{k}} \frac{\partial v}{\partial \vartheta_{k}}
$$

und

$$
\nabla^{2} u=\frac{1}{\sqrt{g}} \sum_{k=1}^{n-1} \frac{\partial}{\partial \vartheta_{k}}\left(\frac{\sqrt{g}}{g_{k}} \frac{\partial u}{\partial \vartheta_{k}}\right) \quad\left(g=\prod_{k=1}^{n-1} g_{k}\right) .
$$

Dann erhalten die Hilfssätze 1 und 2 folgende Form:

Hilfssatz 3. Es sei

$$
S_{1}=\left\{P \mid P \in S, \xi_{1}>0\right\}
$$

und $u=u\left(\xi_{1}, \xi_{2}, \ldots, \xi_{n}\right)$ eine auf $\bar{S}_{1}$ definierte eindeutige zweimal stetig differenzierbare Funktion mit den Eigenschaften:

1) $u\left(\xi_{1}, \xi_{2}, \ldots, \xi_{n}\right) \geqq 0$,

2) $u\left(0, \xi_{2}, \ldots, \xi_{n}\right)=0$.

Dann gilt für jedes endliche $\alpha \geqq 0$

$$
J_{1}(u)=\int_{\bar{S}_{1}}\left(\frac{u}{\cos \vartheta_{1}}\right)^{\alpha-1} u\left((n-1) u+\nabla^{2} u\right) d \omega \leqq 0 .
$$

3) $\nabla^{2} u$ und $(\nabla u)^{2}=(\nabla u, \nabla u)$ sind entsprechend der zweite bzw. der erste Beltramische Operator auf $S_{n}$. Man schreibt bekanntlich auch $\Delta u$ für $\nabla^{2} u$ und $|\operatorname{grad} u|^{2}$ für $(\nabla u)^{2}$. 
Hierbei bedeutet $d \omega$ das Oberflächenelement von S. Das Gleichheitszeichen tritt hier wieder nur für die Funktion $u=C \cos \vartheta_{1}=C \xi_{1}$ ein.

Hilfssatz 4. Ist $v>0$ auf $\bar{S}_{1}$ und dort zweimal stetig differenzierbar, so gilt für jedes endliche $\alpha \geqq 0$

$$
J_{2}(v)=\int_{\bar{s}_{1}}\left(\frac{\cos \vartheta_{1}}{v}\right)^{\alpha+1} v\left((n-1) v+\nabla^{2} v\right) d \omega \geqq 0 .
$$

Auch hier tritt das Gleichheitszeichen dann und nur dann ein, wenn $v=C \xi_{1}$ mit einem konstanten $C$ ist.

Die Beweise beider Sätze verwenden die Identitäten

und

$$
\nabla^{2}(u v)=v \nabla^{2} u+2(\nabla u, \nabla v)+u \nabla^{2} v
$$

$$
\nabla^{2}\left(\cos \vartheta_{1}\right)+(n-1) \cos \vartheta_{1}=0
$$

die zu einer Umformung von $J_{1}(u)$ und $J_{2}(v)$ führen. Man findet in der Tat nach dem Vorbild von 3

$$
J_{1}(u) \leqq-\alpha \int_{\bar{S}_{1}} \sigma^{\alpha-1}(\nabla \sigma)^{2} \cos ^{2} \vartheta_{1} d \omega \leqq 0
$$

und

$$
J_{2}(v) \geqq \alpha \int_{\bar{S}_{1}} \sigma^{\alpha+1}(\nabla \sigma)^{2} \cos ^{2} \vartheta_{1} d \omega \geqq 0
$$

mit $(\nabla \sigma)^{2}=(\nabla \sigma, \nabla \sigma)$ und $\sigma=u / \cos \vartheta_{1}$ bzw. $\sigma^{-1}=v / \cos \vartheta_{1}$. Will man hier das Analogon der Sätze 1 und 2 beweisen, so hat man (unter den entsprechenden Voraussetzungen für Funktionen $u(u \in L)$ bzw. $v(v \in J)$

$$
\mu_{1}(r)=\int_{S_{1}} \sigma_{1}^{2 k} \cos ^{2} \vartheta_{1} d \omega \quad(k \geqq 1)
$$

bzw.

$$
\mu_{2}(r)=\int_{S_{1}} \sigma_{2}^{2 k} \cos ^{2} \vartheta_{2} d \omega \quad\left(k \geqq \frac{1}{2}\right)
$$

mit $\sigma_{1}=u(P) / \cos \vartheta_{1}$ bzw. $\sigma_{2}=\cos \vartheta_{1} / v(P)\left(P=\left(r \xi_{1}, r \xi_{2}, \ldots, r \xi_{n}\right)\right)$.

Es ergeben sich dann leicht die Differentialungleichungen

$$
\frac{\mu_{1}^{\prime \prime}}{\mu_{1}}+\frac{n-1}{r} \frac{\mu_{1}^{\prime}}{\mu_{1}} \geqq \frac{2 k-1}{2 k}\left(\frac{\mu_{1}^{\prime}}{\mu_{1}}\right)^{2}+2 k \frac{n-1}{r^{2}}
$$

und

$$
\frac{\mu_{2}^{\prime \prime}}{\mu_{2}}+\frac{n-1}{r} \frac{\mu_{2}^{\prime}}{\mu_{2}} \geqq \frac{2 k+1}{2 k}\left(\frac{\mu_{2}^{\prime}}{\mu_{2}}\right)^{2}-2 k \frac{n-1}{r^{2}},
$$


die entsprechend durch die Ansätze

$$
\mu_{1}=\left(\frac{y_{1}}{r^{n-1}}\right)^{2 k} \text { bzw. } \quad \mu_{2}=\left(\frac{y_{2}}{r^{n-1}}\right)^{-2 k}
$$

zu den Differentialungleichungen

$$
\frac{y_{1}^{\prime \prime}}{y_{1}}-\frac{n-1}{r} \frac{y_{1}^{\prime}}{y_{1}} \geqq 0
$$

bzw.

$$
\frac{y_{2}^{\prime \prime}}{y_{2}}-\frac{n-1}{r} \frac{y_{2}^{\prime}}{y_{2}} \leqq 0
$$

führen. Somit ist $\left(\frac{d y_{1}}{d r^{n}}\right)^{\prime} \geqq 0$ und $\left(\frac{d y_{2}}{d r^{n}}\right)^{\prime} \leqq 0$. Diese Überlegungen (zunächst auf zweimal stetig differenzierbare subharmonische bzw. superharmonische Funktionen angewandt) führen durch Grenzübergang zu der Konvexität von $r^{n-1} \mu_{2}(r)^{1 /(2 k)} \quad$ bzw. zu der Konkavität von $r^{n-1} \mu_{2}(r)^{1 /(2 k)}$ für jedes $u \in L$ bzw. $v \in J$ als Funktion von $r^{n}$. Nimmt man also die Approximationssätze von subharmonischen bzw. superharmonischen Funktionen durch Folgen von zweimal stetig differenzierbaren subharmonischen bzw. superharmonischen Funktionen zu Hilfe, so hat man die Sätze:

Satz 7. Es sei $u(P)$ subharmonisch nicht negativ in $H: x_{1}>0$ und genüge dort der Bedingung

$$
\varlimsup_{P \rightarrow Q} u(P)=0
$$

für jeden im Endlichen gelegenen Randpunkt $Q$ von $H$. Man setze

$$
L_{k}(r, u)=\left\{\int_{S_{1}}\left(\frac{u}{r \xi_{1}}\right)^{2 k} \xi_{1}^{2} d \omega\right\}^{\frac{1}{2 k}} \quad\left(k \geqq 1 \text { bzw. } k=\frac{1}{2}\right)
$$

mit $u=u\left(r \xi_{1}, \ldots, r \xi_{n}\right)(0<r<+\infty)$. Dann ist $r^{n} L_{k}(r, u)$ konvex in $r^{n}$.

Satz 8. Es sei $v(P)$ superharmonisch und positiv in $H$. Man setze

$$
J_{k}(r, v)=\left\{\int_{S_{1}}\left(\frac{r \xi_{1}}{v}\right)^{2 k} \xi_{1}^{2} d \omega\right\}^{-\frac{1}{2 k}} \quad\left(k \geqq \frac{1}{2}\right) .
$$

Dann ist $r^{n} J_{k}(r, v)$ konkav in $r^{n}$.

Mit Rücksicht darauf, dass $u$ und $v$ nicht negativ sind, kann Satz 8 aus Satz 6 nicht ohne weiteres (durch Betrachtung von - $-u$ ) abgeleitet werden. Lediglich der Grenzfall $k=+\infty$ lässt sich einfach (durch den 
Kunstgriff von 6) behandeln und führt (ohne die Bedingungen $u \geqq 0$, $v \geqq 0$ ) zu zwei dualen Sätzen über die Monotonie von $L(r, u)$ bzw. $J(r, v)$ sowie die Konvexität von $r^{n} L(r, v)$ bzw. die Konkavität von $r^{n} J(r, v)$ als Funktion von $r^{n}$.

Dabei ist hier

$$
L(r, u)=\sup \left\{\frac{u\left(r \xi_{1}, \ldots, r \xi_{n}\right)}{r \xi_{1}} \mid \xi_{1} \in S_{1}\right\}
$$

und

$$
J(r, v)=\inf \left\{\frac{v\left(r \xi_{1}, \ldots, r \xi_{n}\right)}{r \xi_{1}} \mid \xi_{1} \in S_{2}\right\} .
$$

Der interessierte Leser findet den ausführlichen Beweis in meiner Note [8]. Auch die Monotonie von $L(r, u)$ bzw. $J(r, v)$ lässt sich unter Heranziehung der entsprechenden Majoranten (bzw. Minoranten) $H(z)$ von $u$ (bzw. $v$ ) direkt beweisen.

10. Eine Verschärfung des Satzes 3. Ist die partielle Differentialgleichung

$$
\nabla^{2} u=c(z) u \quad\left(\nabla^{2}=\frac{\partial^{2}}{\partial x^{2}}+\frac{\partial^{2}}{\partial y^{2}}\right)
$$

mit einem reellen, stetigen, nichtnegativen $c(z)$ konform invariant, so besitzt diese (nach L. Myrberg [11]) in jedem Gebiet $D$ der komplexen Ebene, dessen Rand $\Gamma$ aus endlich vielen analytischen Kurvenstücken besteht [10], eine Greensche Funktion $G\left(z, z_{0}\right)$ mit der Eigenschaft, dass jede Lösung von (10.1) durch die Gleichung

$$
u(z)=\frac{1}{2 \pi} \int_{\Gamma} u(\zeta) \frac{\partial}{\partial n} G(\zeta, z) d s
$$

gegeben wird. Dabei bedeutet $d s$ das Bogenelement von $\Gamma$ und $\partial / \partial n$ die Differentiation nach der inneren Normalrichtung von $\Gamma$ in $\zeta \in \Gamma$.

Die Funktion $G\left(z, z_{0}\right)$ hat folgende Eigenschaften:

1) $G\left(z, z_{0}\right)$ (kurz $G$ ) ist in $D \backslash\left\{z_{0}\right\}$ regulär und positiv.

2) Es gilt $\nabla^{2} G=c(z) G \quad\left(z \in D \backslash\left\{z_{0}\right\}\right)$.

3) Es ist $\lim G\left(z, z_{0}\right)=0 \quad(\zeta \in \Gamma)$.

4) $G\left(z, z_{0}\right) \stackrel{x \rightarrow \zeta}{+} \log \left|z-z_{0}\right|$ ist beschränkt in der Umgebung von $z_{0}$. Man kehre nun zu den Betrachtungen von 6 zurück und betrachte eine in

$$
H_{0}: 0<R_{1}<|z|<R_{2}<+\infty, \operatorname{Re} z>0
$$


eindeutige zweimal stetig differenzierbare Funktion $u(z)$, die folgenden Bedingungen genügt:

1. $\varlimsup_{z \rightarrow i \eta} u(z) \leqq 0 \quad\left(R_{1}<|\eta|<R_{2}\right)$.

2. In jedem Punkt $z$ von $H_{0}$ ist

$$
\nabla^{2} u \geqq \frac{q^{2}-1}{r^{2}} u
$$

mit einem konstanten $q \geqq 1$.

Schreibt man die rechte Seite der Differentialgleichung

$$
\nabla^{2} v=\frac{q^{2}-1}{r^{2}} v
$$

in der Form

$$
\left(q^{2}-1\right)\left|\frac{d}{d z} \log z\right|^{2}
$$

so schliesst man ohne weiteres daraus, dass (10.4) konform invariant ist, d.h. dass sie bei der konformen Abbildung $z \rightarrow z(\zeta)$ in die Gleichung

$$
\left(\frac{\partial^{2}}{\partial \xi^{2}}+\frac{\partial^{2}}{\partial \eta^{2}}\right) v=\left(q^{2}-1\right)\left|\frac{d}{d \zeta} \log z(\zeta)\right|^{2} v \quad(\xi+i \eta=\zeta)
$$

übergeht. Somit besitzt (10.4) eine Greensche Funktion $G(\zeta, z)\left(z \in H_{0}\right.$, $\zeta \in \Gamma$ ), welche die Eigenschaften 1)-3) hat.

Man definiere nun die Grössen $A_{1}, A_{2}$ durch die Gleichungen

$$
A_{1}=\frac{1}{2 \pi} \int_{\Gamma_{1}} \cos \vartheta \frac{\partial}{\partial n} G(\zeta, z) d s
$$

bzw.

$$
A_{2}=\frac{1}{2 \pi} \int_{\Gamma_{\mathrm{z}}} \cos \vartheta \frac{\partial}{\partial n} G(\zeta, z) d s
$$

und beachte, dass (10.4) in $H$ die beiden Lösungen $v_{1}(z)=r^{\varphi} \cos \varphi$ und $v_{2}(z)=r^{-q} \cos \varphi\left(z=r e^{i \varphi}\right)$ besitzt. Dann wird

und mithin

$$
\begin{aligned}
& r_{1}^{q} A_{1}+r_{2}^{q} A_{2}=r^{q} \cos \varphi, \\
& r_{1}^{-q} A_{1}+r_{2}^{-q} A_{2}=r^{-q} \cos \varphi
\end{aligned}
$$

$$
A_{1}=\frac{r_{2}^{2 q}-r^{2 q}}{r_{2}^{2 q}-r_{1}^{2 q}} \frac{r_{1}^{q}}{r^{q}} \cos \varphi
$$


und

$$
A_{2}=\frac{r^{2 q}-r_{1}^{2 q}}{r_{2}^{2 q}-r_{1}^{2 q}} \frac{r_{2}^{q}}{r^{q}} \cos \varphi
$$

Man bilde jetzt bei gegebenem $u$ mit den Eigenschaften 1) und (10.3) die Grösse

$$
L_{q}(r, u)=\sup \left\{\frac{u\left(r e^{i \vartheta}\right)}{r^{q} \cos \vartheta}|| \vartheta \mid<\frac{\pi}{2}\right\}
$$

für alle $r$ aus dem offenen Intervall $r_{1}<r<r_{2}$. Dass diese Grösse endlich ist, folgt ohne Schwierigkeit aus einem Ergebnis von L. Myrberg [11] (man vgl. auch L. Myrberg [12]), wonach $u(z)$ in jedem Halbkreis $H_{R}:|z|<R$ nicht grösser als die harmonische Funktion sein kann, die auf dem Rand des Halbkreises $H_{R}$ dieselben Randwerte wie $\stackrel{+}{u}(z)$ besitzt. Ist dieses Ergebnis gesichert, so führt die für $u(z)$ gültige Abschätzung

$$
u(z) \leqq \frac{1}{2 \pi} \int_{\Gamma_{1}} u(\zeta) \frac{\partial}{\partial n} G d s+\frac{1}{2 \pi} \int_{\Gamma_{\mathbf{z}}} u(\zeta) \frac{\partial}{\partial n} G d s
$$

zu der Ungleichung

$$
r^{q} \frac{u\left(r e^{i \vartheta}\right)}{\cos \vartheta} \leqq r_{1}^{2 q} L_{q}\left(r_{1}, u\right) \frac{r_{2}^{2 q}-r^{2 q}}{r_{2}^{2 q}-r_{1}^{2 q}}+r_{2}^{2 q} L_{q}\left(r_{2}, u\right) \frac{r^{2 q}-r_{1}^{2 q}}{r_{2}^{2 q}-r_{1}^{2 q}}
$$

(für je zwei Werte $r_{1}, r_{2}, R_{1}<r_{1}<r_{2}<R_{2}$ ), aus der die Konvexität von $r^{2 q} L_{q}(r, u)$ als Funktion von $r^{2 q}$ im Intervall $R_{1}<r<R_{2}$ unmittelbar geschlossen werden kann.

Man kann (nach L. Myrberg) die Ungleichung (10.3) durch den Begriff subelliptisch (in Bezug auf (10.4)) ersetzen. Darunter wird folgendes verstanden: Es sei $D$ ein Gebiet $z \in D$ und es sei $G_{r}(\zeta, z)$ die Greensche Funktion von (10.4) für die offene Kreisscheibe $K_{r}:|\zeta-z|<r$. Gilt dann für alle hinreichend kleine $r$

$$
u(z) \leqq \frac{1}{2 \pi} \int_{\Gamma,} u(\zeta) \frac{\partial}{\partial n} G_{r}(\zeta, z) d s
$$

(wobei $\Gamma_{r}$ den Rand von $K_{r}$ bedeutet), so soll (L. Myrberg [11], [12]) $u(z)$ in Bezug auf (10.4) subelliptisch heissen. Da für eine zweimal stetig differenzierbare Funktion $u(z)$, die (10.3) genügt, die Ungleichung (10.8) mit Rücksicht auf die Greensche Formel richtig ist und umgekehrt (nach L. Myrberg) jede im Sinne von (10.8) subelliptische Funktion in einer kompakten Teilmenge mit stückweise analytischem Rand durch die harmonische 
Majorante von $\stackrel{+}{u}(z)$ abgeschätzt werden kann, so kann das Hauptergebnis dieser Nummer folgendermassen formuliert werden:

Satz 9. Die Funktion $u(z)$ sei in einem Halbring $H_{0}$ :

$$
0<R_{1}<|z|<R_{2}<+\infty, \quad \operatorname{Re} z>0
$$

(in Bezug auf (10.4)) subelliptisch und genüge auf den geradlinigen Begrenzungen von $H_{0}$ der Bedingung 1). Dann ist die Funktion $r^{2 q} L_{q}(r, u)$ im Intervall $R_{1}<r<R_{2}$ konvex in $r^{2 q}$.

11. Heranziehung der Mittelwerte. Ist die Menge

$$
E=\{z \mid u(z)>0\}
$$

für $r>r_{0}$ nicht leer, so liefert die Methode von 4 (angewandt auf die subelliptische Funktion $u(z))$ die Differentialungleichung

$$
\frac{\mu^{\prime \prime}}{\mu}+\frac{\mu^{\prime}}{r \mu}-\frac{2 k-1}{2 k}\left(\frac{\mu^{\prime}}{\mu}\right)^{2}-\frac{2 k}{r^{2}}-\frac{2 k\left(q^{2}-1\right)}{r^{2}} \geqq 0,
$$

wobei wieder $\mu$ durch (4.3) gegeben wird. Somit wird

$$
\frac{\mu^{\prime \prime}}{\mu}+\frac{\mu^{\prime}}{r \mu}-\frac{2 k-1}{2 k}\left(\frac{\mu^{\prime}}{\mu}\right)^{2}-\frac{2 k q^{2}}{r^{2}} \geqq 0
$$

also auch

$$
y^{\prime \prime}-\frac{2 q-1}{r} y^{\prime} \geqq 0
$$

mit

Daraus folgt wieder

$$
y=r^{q} \mu^{1 /(2 k)}
$$

$$
\frac{d}{d r}\left(\frac{d y}{d r^{2 q}}\right) \geqq 0
$$

und mithin die Konvexität von

$$
r^{2 q}\left\{\int_{-\frac{\pi}{2}}^{\frac{\pi}{2}}\left(\frac{\stackrel{+}{u}\left(r e^{i \vartheta}\right)}{r^{q} \cos \vartheta}\right)^{2 k} \cos ^{2} \vartheta d \vartheta\right\}^{\frac{1}{2 k}}
$$

im Intervall $R_{1}<r<R_{2}$ als Funktion von $r^{2 q}$. Ähnlich wie im Falle $q=1$ lässt sich der Satz 8 (eventuell durch Betrachtung der Funktion $u(z)+C r^{q} \cos \vartheta$ mit einem geeigneten konstanten $C$ ) aus der Konvexität der Ausdrücke (11.2) durch Grenzübergang $k \rightarrow+\infty$ ableiten. 
Die Entwicklungen der beiden letzten Nummern können dazu dienen, Aussagen über das Anwachsen von (nicht durchweg nichtpositiven) Lösungen der Differentialgleichung

$$
\nabla^{2} u=c(z) u \quad(c(z) \geqq 0)
$$

in $H$ nach unten abzugrenzen, sofern $u(z)$ der Lindelöfschen Bedingung (10.3) genügt und $c(z)(z \in H)$ ein derartiges Wachstum aufweist, dass

$$
\sigma=\varlimsup_{r \rightarrow+\infty}\left\{r^{2} \inf _{|\vartheta|<\pi / 2} c\left(r e^{i \vartheta}\right)\right\}<q^{2}-1
$$

(mit einem $q>1$ ) ist. Setzt man

$$
M(r, u)=\sup \left\{u\left(r e^{i \vartheta}\right)|| \vartheta \mid<\frac{\pi}{2}\right\},
$$

so liefert die Betrachtung der Grössen (11.2) (insbesondere der mit $k=1$ ) den Satz, dass

$$
\lim _{r \rightarrow+\infty} \frac{M(r, u)}{r^{q}}>0
$$

ausfällt. Ist $\sigma=+\infty$, so kann es keine Funktionen $u(z)$ von endlicher Ordnung geben, die in $H$ l) nicht durchweg nichtpositiv sind, 2) der Bedingung 1 und 3) der Differentialgleichung (11.3) genügen.

Die Theorie der subelliptischen Funktionen (im Sinne der Entwicklungen der beiden letzten Nummern) kann ohne grosse Schwierigkeit auf das entsprechende Problem des $n$-dimensionalen Halbraumes ausgedehnt werden. Dass man hier ebenfalls Juliasche Sätze aufstellen kann, wird der Leser ohne weiteres einsehen. Ebenfalls ist es leicht, die Ergebnisse, die hier gewonnen wurden (für $n=2$ ), durch konforme Abbildung auf allgemeinere, Lindelöfsche Bereiche zu übertragen, wie ich es für den Fall $q=1$ in meiner Arbeit [8] getan habe. Den besten Zugang zu allen Fragen, die dem Denjoy-Carleman-Ahlforsschen Gedankenkreis entspringen, scheint die Methode der Mittelwerte (deren Anfänge auf Carleman zurückgehen) zu bieten.

Freie Universität Berlin

Deutschland 


\section{Literatur}

[1] Ahlfors, L. V.: On Phragmén-Lindelöf principle. - Trans. Amer. Math. Soc. 41,1937, S. $1-8$.

[2] Beckenbach, E. F., und T. Radó: Subbharmonic functions and minimal surfaces. - Trans. Amer. Math. Soc. 35, 1933, S. 648-661.

[3] Blaschke, W.: Kreis und Kugel. - Verlag von Veit \& Comp., Leipzig, 1916.

[4] Dinghas, A.: Über das Phragmén-Lindelöfsche Prinzip und den Julia-Carathéodoryschen Satz. - S.-B. Preuss. Akad. Wiss. 1938, S. 32-48.

[5] -»- Über das Phragmén-Lindelöfsche Prinzip und einige andere verwandte Sätze. - S.-B. Preuss. Akad. Wiss. 1938, S. 123-140.

[6] - - Konvexitätseigenschaften von Mittelwerten harmonischer und verwandter Funktionen. - Math. Z. 63, 1955, S. 109-132.

[7] -»- Über eine Vertiefung des Phragmén-Lindelöfschen Prinzips. - Norske Vid. Selsk. Forh. Trondheim 35:1, 1962.

[8] - - Über eine Fassung des Phragmén-Lindelöfschen Prinzips. - Ann. Acad. Scient. Fennicæ A. I. 319, 1962.

[9] Hayman, W. K.: Questions of regularity connected with the Phragmén-Lindelöf principle. - J. Math. Pures Appl. (9) 35, 1956, S. 115-126.

[10] Myrberg, L.: Über die Integration der Differentialgleichung $\Delta u=c(P) u$ auf offenen Riemannschen Flächen. - Math. Scand. 2, 1954, S. 142-152.

[11] -»- Über die Existenz der Greenschen Funktion der Gleichung $\Delta u=c(P) u$ auf Riemannschen Flächen. - Ann. Acad. Scient. Fennicæ A. I. 170, 1954.

[12] -»- Über subelliptische Funktionen. - Ann. Acad. Scient. Fennicæ A. I. 290, 1960.

[13] Nevanlinna, F. und R.: Über die Eigenschaften analytischer Funktionen in der Umgebung einer singulären Stelle oder Linie. - Acta Soc. Sci. Fenn. 50: 5, 1922.

[14] TsujI, M.: Potential theory in modern function theory. - Maruzen Co., Ltd., Tokyo, 1959. 